\title{
Evaluation of User Interface Design and Input Methods for Applications on Mobile Touch Screen Devices
}

\author{
Florence Balagtas-Fernandez, Jenny Forrai, and Heinrich Hussmann \\ Media Informatics Group, Department of Computer Science, \\ University of Munich, Germany \\ florence.balagtas@ifi.lmu.de, forrai@cip.ifi.lmu.de, \\ heinrich.hussmann@ifi.lmu.de
}

\begin{abstract}
With the advent of touch screen phones, good UI design and simplified input methods for applications running on such devices are important factors that contribute to its popularity and success. The goal of this research is to evaluate different user interface designs and input methods for mobile phones with touch screen capability. In order to do this, two prototypes of a simple social networking application were implemented using a G1 phone that runs the Android platform. A user study has been conducted to compare different input techniques and UI designs and evaluate their usability.
\end{abstract}

\section{Introduction and Motivation}

Mobile phones with advanced capabilities often employ touch screen as one of the main interaction methods. By adding touch screen capability, it is easier for users to carry out certain actions depending on the task they want to accomplish. However, even with the emergence of such technologies and input capabilities, the design of applications running on such devices is critical for the success of both the device and the application. Usability still plays a big role in its acceptance in the mobile market. It may be difficult to change the design of the actual hardware itself, but a welldesigned application can be a big help in order to overcome such limitations.

Weiss [1] provided a list of guidelines for designing user-interfaces for handheld devices. However, these guidelines are very general and do not have concrete examples of UI components to be used based on specific models of handheld devices. Oehl et al. [2] looked into the correlation between the size of a screen's display to the difficulty of the pointing task influenced by the size of the target for touch screen displays using a stylus pen. This differs from our research since instead of evaluating the sizes of target components on the screen, we focus on the actual UI components that should be used in order to easily input information. The investigation done by Hoggan et al. [3] regarding the interaction with mobile devices without physical keyboards resulted in the conclusion that performance improved when tactile feedback is involved.

The research reported here differs from the mentioned existing researches in a way that we would like to focus on investigating which specific UI components promote ease-of-use. The three factors investigated were the overall interface layout, information input and menu accessibility. In particular, the focus of the work was on comparing a scrollable view versus a tabbed view for the layout, direct input using a 
keyboard versus input by tapping on the screen through a modal dialog, and menu accessibility through the device menu versus the context menu. The G1 phone that runs the Android platform was used as the sample device for this research.

\section{BDroid Protoype Designs}

For the purpose of this study, an application called BDroid was developed. BDroid is a social networking application similar to its web-based counterparts such as Facebook and LinkedIn. Features of BDroid includes: an editable user profile, friends list with links to other friends' profile, sending/receiving messages, ability to share pictures, calendar and a search function to look for other registered BDroid users. Two versions of BDroid were developed, each featuring a different kind of user interface design and input methods. Fig. 1 describes the different layouts, input methods and menus used for both prototypes.

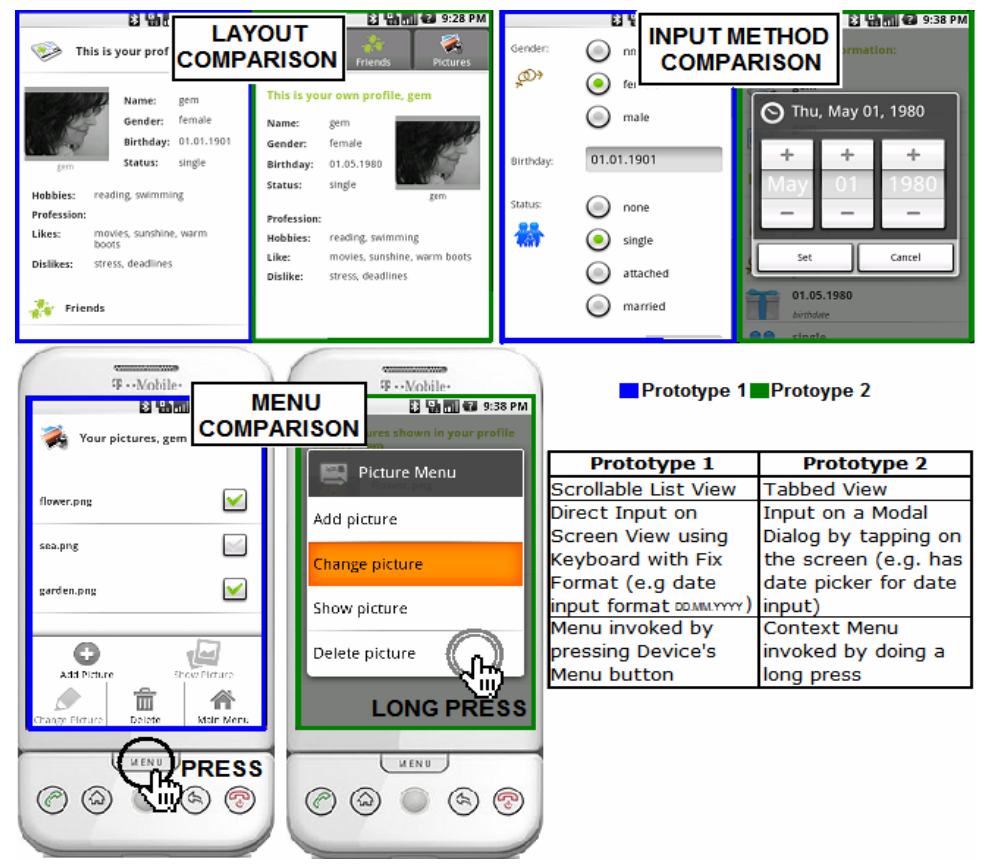

Fig. 1. BDroid prototypes and feature comparison

\section{User Study Evaluation and Results}

Ten people participated in the user study whose ages ranged from 23 to 53 years old ( $40 \%$ female, $60 \%$ male). All of the participants own a mobile device, and the average length of ownership is nine years. Out of the ten participants, only $10 \%$ own a mobile device with touch screen capability. In terms of experience with mobile device usage, 
$40 \%$ of the participants claim to have very good experience in using mobile devices, while $30 \%$ only rated themselves average and the rest said they are inexperienced with mobile device usage. The design of the user study was a within-subjects factorial design. The order in which the two prototypes were used was counterbalanced to minimize learning effects.

In the beginning of the user study, the participants were briefly taught how to use the phone such as accessing the context menu by doing a long press on the screen, changing the orientation of the phone to view the screen in landscape or portrait view, using the hardware components (e.g. keyboard, device menu, trackball), etc. The independent variables were the two different versions of the prototypes, which differed in terms of the layout of components (tabs vs. list view) and information input (keyboard input on non-modal screen vs. tapping on modal screen, context menu vs. device menu). Task times were measured through a logging system within the BDroid prototypes. Qualitative information was also collected in a form of a questionnaire at the end of each set of tasks.

The Tasks. Each participant was asked to perform specific tasks for each prototype of the BDroid application. For both prototypes, each task can be optimally accomplished in the same number of steps and basically differ in terms of how the user interface components are displayed and which components are used for input. The subtasks that we concentrated on looking into and the specific factors evaluated are: navigating from the main profile to the edit profile section (evaluate layout), editing profile (evaluate input methods) and, searching and adding/viewing a user (evaluate menu accessibility).

Results, Analysis and Empirical Observations. For the Navigate task, the average time to navigate using the scrollable view was significantly faster $(\mathrm{M}=16.40 \mathrm{sec}$ onds, $\mathrm{SE}=2.56)$ as compared to the tabbed view $(\mathrm{M}=42.80$ seconds $\mathrm{SE}=13.58$, $\mathrm{t}(9)=-1.868, \mathrm{p}<0.05)$. This correlates to the users' preference in which $80 \%$ of them said it was easier to navigate using the scroll view as compared to the tabbed view. However, in terms of layout, $80 \%$ chose the tabbed view.

Through our empirical observation of how the users interacted with the prototypes, the probable reason why it was easier for them to navigate using the scrollable view was that, most of them used the trackball on the device. When the user was grasping the device with both hands when in horizontal position, there was no need to release their hold on the device since the trackball was easily accessible with the thumb. In contrast to navigating with the tabbed view, the user had to move the dominant hand in order to tap the tabs on the screen, or move one of the fingers closer to the screen which was relatively unreachable as compared to the trackball especially when both hands were grasping the device. An exception to easy navigation using the scroll view was when the user tried to scroll using the onscreen scrollbar by dragging their fingers on the screen. The problem with the onscreen scrollbar was that, it was too thin and tends to be occluded for users with large fingers. Another problem which was more hardware oriented was that, the screen itself was not too responsive to the touch.

For the Edit task, using the keyboard was faster $(\mathrm{M}=108.30$ seconds, $\mathrm{SE}=17.57)$ compared to inputting information by tapping on the screen $(M=130.00$ seconds, $\mathrm{SE}=20.72$ ). Again, problems encountered were similar to the reasons previously mentioned which was basically influenced by the insensitivity of the screen to touch. 
Other probable reasons why keyboard entry was faster was that, it uses the common QWERTY keyboard layout and also the tactile feedback [3] it provided. Changing the orientation of the device horizontally every time a user inputs something also proved to be time consuming and inconvenient.

For the Search and Add/View task, using the device menu was faster ( $\mathrm{M}=48.40$ seconds, $\mathrm{SE}=14.04)$ as compared to the one using the context menu $(\mathrm{M}=79.40$ seconds, $\mathrm{SE}=23.04)$. Accessing the context menu via long press was also counterintuitive which most users mentioned in the post-test questionnaire.

For the last two tasks mentioned, the average times in which the two prototypes differ were not statistically significant. However, it can be concluded that most users preferred the prototype that allowed the tasks to be carried out faster as seen in Fig. 2.
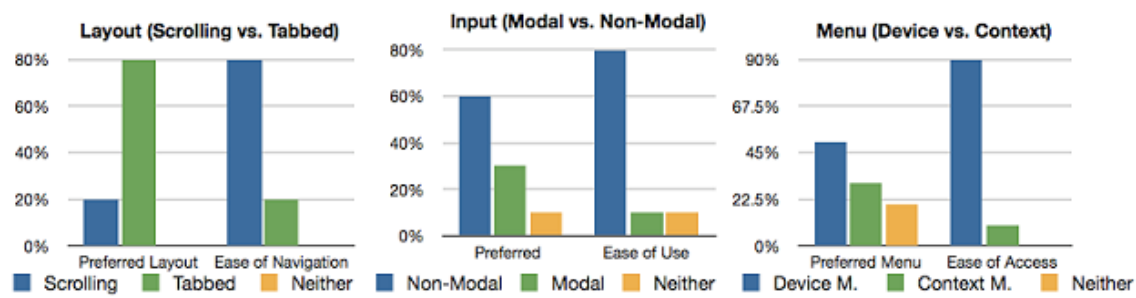

Fig. 2. Qualitative results of the post-test questionnaire

\section{Summary and Conclusion}

Two different prototypes that feature different styles of UI and input methods for mobile devices with touch screen capability were presented. Evaluation was done in order to see which design provided the user better ease-of-use. However, the user interface components and input methods described in this paper are just part of a bigger work that should be done to create a complete catalog of comparisons of different UI controls that promote usability. Automated tool support which gives the developer assistance on which specific UI elements to use with their applications would also be useful. It should be noted that, even though general design guidelines are helpful, specific examples that applies these guidelines for a specialized range of devices (e.g. using specific UI components for devices with touch screen capability) is more beneficial for the developers and designers whose goal is to create beautiful and usable applications.

\section{References}

1. Weiss, S.: Handheld Usability. John Wiley and Sons Ltd., Chichester (2002)

2. Oehl, M., Sutter, C., Ziefle, M.: Considerations on efficient touch interfaces - how display size influences the performance in an applied pointing task. In: Smith, M.J., Salvendy, G. (eds.) HCII 2007. LNCS, vol. 4557, pp. 136-143. Springer, Heidelberg (2007)

3. Hoggan, E., Brewster, S.A., Johnston, J.: Investigating the effectiveness of tactile feedback for mobile touchscreens. In: Proceeding of the twenty-sixth annual SIGCHI conference on Human factors in computing systems. ACM, Florence (2008) 